\title{
Bio-Inspired Algorithms for Color Image Segmentation
}

\author{
Salima Nebti \\ Department of computer science \\ FerhatAbass university \\ Algeria, Sétif 19000
}

\begin{abstract}
Effective image segmentation remains a challenging process as it constitutes a critical step to higher level image processing applications such as pattern recognition. In this paper, we present bio-inspired formulation to perform unsupervised image segmentation. Specifically, we used the Quantum PSO, the hybrid Gravitational PSO algorithm, a cooperative gravitational approach and the bees approach as powerful global classifiers to optimize the partition of image data into homogenous regions. The segmentation accuracy based on the bees' algorithm has the highest accuracy.
\end{abstract}

\section{Keywords}

Image segmentation, Quantum PSO, the Gravitational search algorithm, cooperative coevolution, the bees algorithm.

\section{INTRODUCTION}

Image segmentation is one of the most important challenges encountered in automatic image processing and it is crucial for image understanding and analysis to interpret its contents. Tremendous research has been devoted to image segmentation but remains an active research topic as it is the basic step in a wide range of applications such as medicine, Remote Sensing, content based image retrieval, object tracking....etc.

In general, image segmentation is a hard task which refers to automatic decision of homogenous regions. The output of image segmentation may be either homogenous regions or, detected contours or any other information which locates an object in object tracking applications.

Several segmentation algorithms are obtainable that can be classified into four groups [1]: Classification or thresholding, region-based segmentation, contour based segmentation and the hybrid approach which is a mixture of the two previous methods.

Classification is a frequently used solution to achieve image segmentation. Grey level, color and texture are the most extracted features for pixels classification. These features are used as the basis for a similarity measurement between image pixels. Classification methods can be divided in two families, according to whether they analyze the distribution of pixel colors or the coordinates of similar colors. However, the classification methods are most often applied to pixel values, regardless to the spatial information. Some of recent works on pixel based image classification can be found in [2], [3], [4]

The region-based segmentation can be divided in two types: region-growing and split and merge. The region growing algorithms start from a set of seed regions, which can be found automatically or provided by the user, the regions then grow by incorporating the most similar pixels according to a given criterion while split and merge algorithms are based on the principle of grouping similar pixels into homogenous regions and subdividing the heterogeneous regions until satisfying an homogeneity criterion.
Contour based methods such as deformable models and active contours are used to detect regions of interest by placing contours between heterogeneous regions. The hybrid approach is a cooperation between region and contour based segmentations and therefore to improve accuracy [5].

The concern of this paper is the use of efficient bio-inspired optimization algorithms for unsupervised colour image classification. Specifically we used quantum PSO, the gravitational search algorithm and the bees algorithm for color image segmentation, we have applied these methods to segment satellite as well as brain images and found the results very encouraging.

The originality of this work, mainly relates to image clustering in a distributed way based on new dynamics used to solve and judge the best quality of segmentation. The main motivation behind these new swarming optimization methods is to benefit from the parallel that allow using distributed agents over the search space in order to find the best solution, in addition swarming methods ensure minimal costs in accordance with maximum effectiveness.

The organization of this paper is as follows. In section 1, the most prominent related works are provided. In section 2 , the Quantum PSO, GSA, cooperative GSA-PSO and BA are explained, and then we begin by evoking how unsupervised image segmentation is solved through the aforementioned algorithms in sections 3,4 and 5. Next, the obtained results with the suggested methods are shown and discussed in section 6. Finally, section 7 concludes with some final remarks.

\subsection{Previous work}

Abundant literature has been investigated by many authors on methods used for image segmentation such as thresholding based Models, Neural networks [6],[7],Markov Random Field [8] , Multi- resolution [9], Watershed and Wavelet models. An extensive review on these models for remotely sensed images can be found in [10] and for medical images can be found in [11].

Recently, the use of optimization methods to improve image segmentation has been drastically increased. Various methods have been proposed for this purpose, many of them focus on the use of ant colony optimization and particle swarm optimization, and some of these are:

In the work presented in [12], PSO, the Chaos Particle Swarm Optimization (CPSO) and artificial bees colony optimization $(\mathrm{ABC})$ were used to find the optimal threshold of a 2-D histogram segmentation method. Experiments showed that the CPSO based approach has the best performance. In [13], results indicated that ant colony optimization and PSO can improve the K-means and the simple competitive learning SCL algorithm for image segmentation. In [14], the speed of the Fuzzy C-means (FCM) image segmentation was enhanced and its accuracy was improved based on PSO as FCM centers 
optimizer. This work [15] proved that the ABC-based image segmentation has the best results compared to K-means, FCM and PSO-based segmentation. This paper [16] proved the efficiency of particle swarm optimization (PSO) as a new multilevel thresholding method for image segmentation. In this work [17], an ant colony-based edge detector has been suggested that combines a nonlinear contrast enhancement, called Multiscale Adaptive Gain, and the Ant Colony Optimization.

\section{THE USED SEARCH ALGORITHMS}

The interest of this work is the application of QPSO, GSA and the bees algorithm to image segmentation. Therefore the basic explanation of these methods is needed.

\subsection{The Quantum Particle QPSO:}

QPSO is a recently population based algorithm derived from quantum concepts to describe the movement of particles [18], [19]. In PSO method, each particle has its own position and speed. Thus its trajectory into the search space is well defined using recursive equations described in [20]. Like PSO, in QPSO, particles are distributed over the search space searching for optima by updating the set of particles positions. The particularity of QPSO is that the evolution of the population is inspired by PSO and quantum mechanics theories to ensure better diversity. QPSO is based on the atom quantum model, where charged particles (electrons) do not follow a determined trajectory as in PSO method, but are randomly initialized around the best position found by the swarm of particles.

In quantum world, the particle trajectory could not be well defined, since its position and speed could not be observed at the same time due the uncertainty principle of quantum computing. Unlike PSO, the state of particles in QPSO is represented by a wavefunction $\psi(x, t)$, that is the state of particles is probabilistic relying on the following quantum computing rules [21], [22]:

$$
\begin{aligned}
& x_{i}^{d}(t+1)=p_{i}^{d}(t)+\beta\left|\operatorname{Mbest}^{d}(t)-x_{i}^{d}(t)\right| \cdot \ln (1 / \mu), \\
& \text { if } k>0.5 \\
& x_{i}^{d}(t+1)=p_{i}^{d}(t)-\beta \mid \text { Mbest }^{d}(t)-x_{i}^{d}(t) \mid \cdot \ln (1 / \mu), \\
& \text { if } k \leq 0.5
\end{aligned}
$$

Where:

$x_{i}^{d}(t)$ is the position of $\mathrm{i}^{\text {th }}$ particle for the $\mathrm{d}^{\text {th }}$ dimension in the $\mathrm{t}^{\text {th }}$ (iteration)

Mbest $^{d}(t)$ is the mean of the pbest positions of all particles for the $\mathrm{j}^{\text {th }}$ dimension as explained in eq 2

$\beta$ is a contraction-expansion parameter : This parameter controls the balance between exploration and exploitation. A great value of $\beta(>1)$ produces better exploration and a low value of $\beta(<1)$ implies low amplitude of movement and thus better exploitation.

$\mathrm{u}$ and $\mathrm{k}$ are uniformly distributed values within $[0,1]$

$$
\operatorname{Mbest}^{d}(t)=\frac{1}{N} \sum_{d=1}^{d i m} p_{i}^{d}(t)(2)
$$

$\mathrm{N}$ represents the number of particles.

$\mathrm{pi}(\mathrm{t})$ is a local attractor position used to guarantee convergence as depicted in eq 3

$$
p_{i}^{d}(t)=\frac{c_{1} * \text { Pbest }_{i}^{d}+c_{2} * \text { gbest }_{g}^{d}}{c_{1}+c_{2}}
$$

Pbest and gbestare respectively the personal and global best positions, $C_{l}$ and $C_{2}$ : are respectively the cognitive and social factors.

Various other new and better versions of QPSO have been presented in the last few years such as the Gaussian QPSO [23].

\subsection{The gravitational search algorithm}

Gravitational search algorithm (GSA) is one of the newest swarm based optimization algorithms [26] which relies on the Newtonian law of gravity and mass interaction. In this algorithm, potential solutions may be seen as distributed agents that have different masses. The movement of these agents is guided by the gravitational magnetism force between them; therefore, agents are attracted towards the agents with heavier masses, that is, towards good solutions [24], [25].

The GSA dynamic is as follows [27], [29]:

This algorithm starts with random solutions $X i$ known as agents:

$X_{i}=\left(x_{i}^{1}, \ldots, x_{i}^{d}, \ldots, x_{i}^{n}\right), \quad i=1,2, \ldots \ldots \ldots s$

Where $x_{i}^{d}$ is the position of $i$ th agent in the $d$ th dimension.

The mass of each agent is evaluated based on its fitness fitness $_{i}(t)$, the best and the worst fitness values in the current iteration $t$ as follows:

$$
\begin{gathered}
Q_{i}(t)=\frac{\operatorname{fitness}_{i}(t)-\operatorname{worst}(t)}{(\operatorname{best}(t)-\operatorname{worst}(t))} \\
\operatorname{Mass}_{i}(t)=Q_{i}(t) / \operatorname{sum}\left(Q_{j}(t)\right)
\end{gathered}
$$

Based on the gravity law, the acting forces that apply the set of agentsj to an agent $i$ are given by Equation 6[27], [29].

$$
\begin{gathered}
\operatorname{force}_{i}^{d}(t)=G(t) \operatorname{mass}_{i}(t) \sum_{j=1, j \neq i}^{s} r_{j} \frac{\operatorname{mass}_{j}(t)}{\operatorname{Dist}_{i j}(t)+\varepsilon}\left(x_{j}^{d}(t)\right. \\
\left.-x_{i}^{d}(t)\right)
\end{gathered}
$$

The agent acceleration derived from the motion law is given by (Equation (7)) where a slit modification has been suggested by Rashedi et al. [26] to allow better exploration and exploitation; this is by limiting the magnetic force to only the $\mathrm{k}$ best found agents[27], [29].

$$
\begin{aligned}
\operatorname{acc}_{i}^{d}(t)=\frac{\operatorname{force}_{i}^{d}(t)}{\operatorname{mass}_{i}(t)} & \\
= & G(t) \sum_{j \in k \text { best }, j \neq i} r_{j} \frac{\operatorname{mass}_{j}(t)}{\operatorname{Dist}_{i j}(t)+\varepsilon}\left(x_{j}^{d}(t)\right. \\
& \left.-x_{i}^{d}(t)\right)(7)
\end{aligned}
$$

Equation (8) is used to compute the next velocity of agent $i$ then equation (9) is applied to evaluate its next position.

$$
\begin{gathered}
\text { vitesse }_{i}^{d}(t+1)=r_{i} \text { vitesse }_{i}^{d}(t)+\operatorname{acc}_{i}^{d}(t)(8) \\
x_{i}^{d}(t+1)=x_{i}^{d}(t)+\text { vitesse }_{i}^{d}(t)(9)
\end{gathered}
$$

Where $r i$ and $r j$ are two uniformly distributed random numbers in the interval $[0,1]$, andęis a small value. $G(t)$, 
called the gravitational constant, is an exponentially or linearly decreased function. $\operatorname{Dist}_{i j}(t)$ is the Euclidean distance between two agents $i$ and $j$.

In our work, we used the hybrid GSA-PSO where some equations were modified as follows [28]:

Equations 4 \&5 by:

$$
\begin{gathered}
Q_{i}(t)=\frac{\operatorname{fitness}_{i}(t)-0.99 * \operatorname{worst}(t)}{(\operatorname{best}(t)-\operatorname{worst}(t))} \\
\operatorname{Mass}_{i}(t)=5 * Q_{i}(t) / \operatorname{sum}\left(Q_{j}(t)\right)
\end{gathered}
$$

Equation (7)by (12):

$$
G(t)=\exp \left(-\operatorname{Dist}_{i j}(t)\right)
$$

We have replaced the gravitational constant by an exponentially varying function that has as argument the distance between the $\mathrm{k}$ best agents and the other agents to avoid local solutions.

$$
\begin{gathered}
\operatorname{acc}_{i}^{d}(t)=0.5 * \exp \left(-\operatorname{Dist}_{i j}(t)\right) \\
* \sum_{\substack{\left.j \in k \text { best }, j \neq i \\
-x_{i}^{d}(t)\right)}} r_{j} \frac{\operatorname{mass}_{j}(t)}{\operatorname{Dist}_{i j}(t)+\varepsilon}\left(x_{j}^{d}(t)\right.
\end{gathered}
$$

and equations (8) and (9) by (13) and (14):

$$
\begin{aligned}
\text { vitesse }_{i}^{d}(t+1)= & r_{i} \text { vitesse }_{i}^{d}(t)+C_{1} r_{i} * \operatorname{acc}_{i}^{d}(t) \\
& +C_{2} r_{i}\left(\text { bestsolution }-x_{i}^{d}(t)\right) \\
x_{i}^{d}(t+1)=x_{i}^{d}(t) & + \text { vitesse }_{i}^{d}(t)
\end{aligned}
$$

\section{PROBLEM FORMULATION}

We have applied the explained algorithms to partition the set of image pixels into a predefined number of regions, that is, how many regions are desired (according to previous knowledge in some applications).

In order to apply the aforementioned algorithms to image segmentation, we first need to specify what are agents, their positions and velocities. We also need to specify a suitable segmentation criterion. The term of agent refers to a potential solution that is a particle in QPSO, a bee in BA or an object in GSA-PSO. In this work, each region is represented by its mean center; a potential solution is a set of centers (regions) selected in iterative way through QPSO, BA GSA-PSO or cooperative GSA-PSO.

A particle (an object) is a vector of $\mathbf{k}$ clusters centers which are randomly initialized as presented below:

\begin{tabular}{|l|l|l|l|l|}
\hline Center $_{1}$ & Center $_{2}$ & $\ldots . . . . .$. & & Center $_{\mathrm{k}}$ \\
\hline
\end{tabular}

Therefore, to find a partition of pixels into $\mathrm{K}$ classes (regions) so that pixels within the same region are similar as possible and pixels belonging to different regions are dissimilar as possible, pixels are grouped based on the sum of squared errors SSE criterion to measure the regions homogeneity; the sum of squared errors is a widely used criterion for data clustering or unsupervised classification. Our algorithms consider each region center as independent entity then iteratively change their positions based on recursive equations and then efficiently select the most relevant regions centers according to the best found value of SSE as agents' fitness.
QPSO, BA GSA-PSO or cooperative GSA-PSO aims to select the set of centers minimizing the SSE criterion given by eq (15)

$$
S S E=\sum_{i=1}^{M} \sum_{x \in C_{i}}^{N}\left\|x-\bar{C}_{i}\right\|^{2}
$$

Where M: is the number of centers

$$
\begin{aligned}
& \mathrm{N}: \text { is the number of pixels per class } \\
& \bar{C}_{i}: \text { are centers of classes }
\end{aligned}
$$

Within the framework of color image segmentation, the used features are pixel colors, thus, each pixel is represented by its three component colors in the RGB space. In order to construct the segmented image, the regions are labeled with the label of their relevant centers distinguished by (dis) similarity through the used SSE homogeneity criterion. The best solution is the centers of clusters representing the most differentiating colorimetric features giving the minimal value of the SSE criterion thanks to the aforementioned optimization methods.

The proposed algorithms are summarized below:

\subsection{The Quantum PSO for image segmentation}

Initialize a random population of particles: each particle is a set of random clusters centers in image space

For a number of iterations do

$\{$ For each particle do

\{Assign each pixel to the nearest center \& evaluate the particle fitness.

Save the personal best (pbest) positions.

Save the global best (gbest) \}

Compute the mean best (Mbest) using eq 2

For each particle do

\{Update particles positions through eqs 1 \}

Show image partition based on pixels labeling to their nearest best centers

\subsection{The Gravitational Search Algorithm for image segmentation}

Initialize a set of $\mathrm{S}$ agents with random positions in image space and random velocities

For a number of iterations do

$\{$ For each agent do

\{Assign each pixel to the nearest agent center \& evaluate its fitness through eq (15)

Evaluate its mass eq (10) and (11)

Compute its acceleration eq (12)

Update its velocity eq (13) 
Update its position eq (14) \}\}

Show image partition based on pixels labeling to their nearest best centers

\subsection{The cooperative GSAPSO for image segmentation}

Image segmentation using the Cooperative GSA-PSO is basically inspired by the work of [30] used to function optimization but applied here to color image segmentation. In general, one classifier for a multi-dimensional problem such as color image segmentation delivers a weak decision on pixels classification, to obtain higher accuracy in terms of regions homogeneity, it is possible to fusion the complimentary decisions which can exist between different color spaces. For that, we combine the obtained decisions of three GSA-PSO based classifiers where each one searches for the best sub-solution in a specific color space in cooperation with the other sub-solutions. This would have intense research in agents' evolution.

This approach consists of three GSA-PSO classifiers where each of them searches for the best sub-solution in a specific color space; these classifiers can be viewed as dissimilar species evolving together in order to solve image segmentation through co-evolution. The fitness of each species is a dependent measure which is computed based on its cooperation with randomly chosen agents from the other species and/or their best found ones to construct a complete solution following the dynamic bellow:

Initialize a population of complete solutions (three color centers)

Apply the GSA-PSO algorithm to find the best complete solution and then send its sub-solutions to the relevant species to evaluate their quality against random sub-solutions in the other color spaces as follows

Initialize three species (populations) with a known number of centers in the three color spaces (RGB).

For a number of iterations do

\{For each species (R, G or B) do

\{Combine the relevant best found sub-solution, as described above, with sub-solutions in the other color spaces (these subsolutions are considered as collaborators in the relevant color space).

Evaluate its fitness using the SSE (15)

Save the best found complete solution

Apply the GSA-PSO to evolve collaborators' positions in the related colour space \}\}.

Show the best found complete solution

\subsection{The Bees based approach}

The Bees Algorithm is an effective bio-inspired algorithm which imitates the foraging behavior of honey bees. The key idea of this algorithm is to accomplish a neighborhood search in accordance with a random search for better exploitation and exploration of the search space. So, a part of bees are specified for searching in the neighborhood of best sites and the rest are designed for random search seeing for better flower sites [31], [32].

The bees algorithm for color image segmentation is as follows [33]:

Bees initialization: a bee is a number of random centers chosen from image space

For $\mathrm{P}=1$ :NumberOfscouts do

\{Assign each pixel to the nearest center\& evaluate the scout quality using eq (15)

Perform an ascending order of scout solutions to determine the elite and site bees $\}$

For a number of iterations do

$\{$ For $\mathrm{p}=1$ :numberOfelite do

For $\mathrm{F}=1$ : aroundelite do

$\{$ Determine a neighboring bee around the current elite bee

Evaluate the elite fitness \& replace it by the current neighboring bee if it is better\}\}.

For $\mathrm{F}=($ aroundelite +1$):$ aroundsite do

\{Determine a neighboring bee around the current site bee

Evaluate the current site fitness \& replace it by the neighboring bee it is better \} .

For $\mathrm{F}=($ aroundsite +1$)$ : NumberOfscout

\{Initialize the rest of scout bees in a random way\}

For $\mathrm{F}=1$ : NumberOfscout

$\{$ Perform an ascending order of all found solutions

Determine the best solution \}\}

Show the best solution by pixels labeling to their nearest centers.

\section{EXPERIMENTS}

To assess the performance quality, many types of images were tested such as remotely sensed and medical images. Results are illustrated in detail and compared.

The initial values of needed parameters are as follows:

Table 1: initial parameters

\begin{tabular}{l|l}
\hline QPSO & population size $=20, \mathrm{Nb}$ of iterations $=50$. \\
\hline GSA & population size $=20, \mathrm{Nb}$ of iterations $=50$ \\
\hline CGSA-PSO & $\begin{array}{l}\text { population size for each species } 20, \mathrm{Nb} \text { of } \\
\text { iterations }=50 .\end{array}$ \\
\hline BA & $\begin{array}{l}\text { The number of scout bees }=100, \text { The number } \\
\text { of elite bees }=40, \text { The number of sites }=40, \\
\text { The number of bees around elite bee }=50, \\
\text { The number of bees around site bee }=10, \text { The } \\
\text { size of neighborhood }=0.0234, \mathrm{Nb} \text { of } \\
\text { iterations }=50 .\end{array}$ \\
\hline
\end{tabular}

During this study, each algorithm has been tested ten runs; the exposed values represent the relevant mean accuracy of each method. 
The best solutions of each algorithm on Fig1 when 4 classes are needed with the resultant graphs behavior are depicted below:

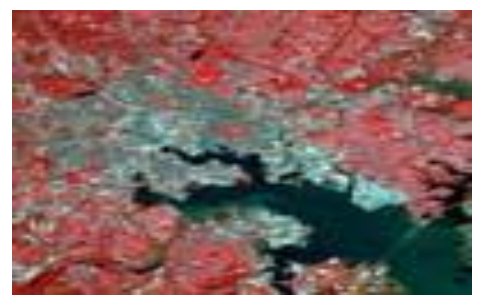

Fig1: original image

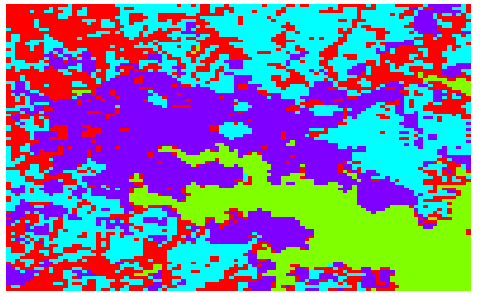

Fig2: QPSO: $\mathrm{SSE}=1.3073 \mathrm{e}+007$

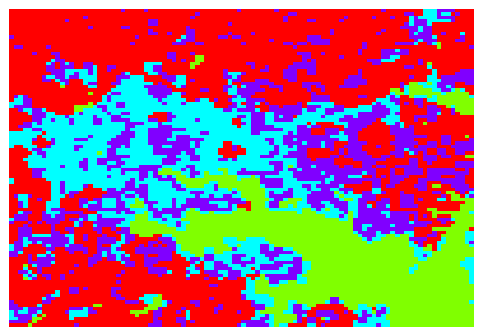

Fig3: GSA-PSO SSE=1.3424e+007

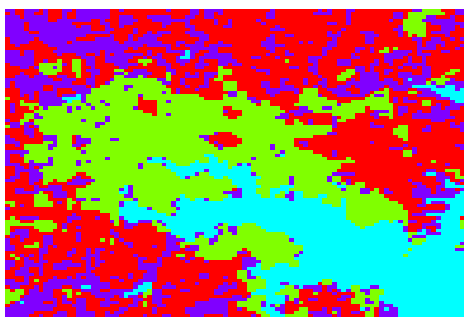

Fig4: CGSA-PSO SSE $=1.3216 \mathrm{e}+007$

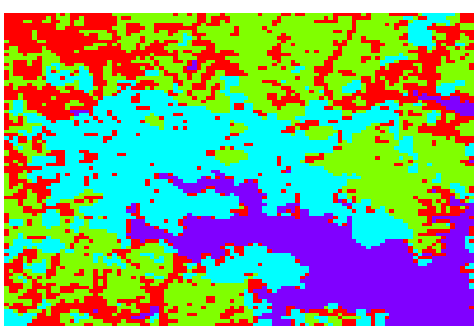

Fig5: $\mathrm{BA}: \mathrm{SSE}=1.3013 \mathrm{e}+007$
Table 2: comparative results

\begin{tabular}{ll}
\hline Algorithm & The Sum of squared error \\
\hline QPSO & $1,3396 \mathrm{e}+007$ \\
GSA-PSO & $1,3692 \mathrm{e}+007$ \\
CGSA-PSO & $1,3552 \mathrm{e}+007$ \\
BA & $1,3021 \mathrm{e}+007$ \\
\hline
\end{tabular}

As these results show, bio inspired algorithms perform well in image segmentation, the Quantum PSO based method provides better results than GSA-PSO and Cooperative GSAPSO. The two previous methods produce lower accuracy than the bees' algorithm based segmentation; its improvement is due to the larger search space provided by the neighborhood search strategy in joint with randomness. Thus, the bees' algorithm is a simple and effective tool for unsupervised image segmentation.

\section{CONCLUSION}

In this paper, the main issues of unsupervised image classification have been addressed, including color clustering and cooperative classifiers. The proposed segmentation algorithms provide a solution for both grey level and color images. During this study, different swarming based optimization methods have been applied to benefit from their inherited parallelism and thus to achieve effective image segmentation. These algorithms are the quantum PSO, the hybrid PSO and gravitational search algorithm, the cooperative GSA-PSO and the bees algorithm. Using these processes, it is possible to circumvent local optima and thus to enhance segmentation quality. Experiments proved that the suggested QPSO based method efficiently outperforms the hybrid GSA-PSO based approach and the cooperative GSAPSO, The BA based image segmentation is able to achieve the best results. These algorithms can be used to improve wider range of image segmentation algorithms such as Markov Random Field based segmentation, Self-organized maps and multi-resolution.

\section{REFERENCES}

[1] Dinesh D. P, Sonal G. D, Medical Image Segmentation: A Review, International Journal of Computer Science and Mobile Computing, IJCSMC, Vol. 2, Issue. 1, January 2013, pg.22 - 27

[2] Rao K.Y, Stephen M.J, Phanindra D.S, Classification Based Image Segmentation Approach, IJCST Vol. 3, Issue 1, ISSN: 2229-4333, 2012.

[3] Chitade A.Z, Katiyar S.K, Colour based image segmentation using K-means clustering, International Journal of Engineering Science and Technology, Vol. 2(10), 2010, 5319-5325. 2010

[4] Dong G, Xie M, Color Clustering and Learning for Image Segmentation Based on Neural Networks, IEEE Transactions on neural networks, Vol. 16, N ${ }^{\circ} .4,2005$.

[5] Cufi X, Munoz X, Freixenet J, Marti J, A Review on Image Segmentation Techniques Integrating Region and Boundary Information. Advances in Imaging and Electronics Physics, 120:1-32, 2002.

[6] Catalin A. A review on neural network-based image segmentation techniques. 2001. 
[7] Moghaddam M. J, Zadeh H.S, Medical Image Segmentation Using Artificial Neural Networks, ISBN: 978-953-307-243-2. 2011.

[8] Ouadfel S, BatoucheM, Ant colony system with local search for Markov random field image segmentation, ICIP (1) 2003: 133-136

[9] Tab, F.A. Naghdy, G., Mertins, A. Scalable Multiresolution Image Segmentation and Its Application in Video Object Extraction Algorithm, Page(s): $1-6$, TENCON 2005.

[10] Dey V, Zhang Y, Zhong M, A Review on Image Segmentation Techniques with Remote Seneing Perspective, ISPRS TC VII Symposium - 100 Years ISPRS, Vienna, Austria, July 5-7, IAPRS, Vol. XXXVIII, Part 7A, 2010

[11] Tripathi S, Kumar K , Singh B.K, Singh R.P, Image Segmentation: A Review, International Journal of Computer Science and Management Research, Vol 1 Issue 4, ISSN 2278-733X, 2012.

[12] Zhiwei Ye, Zhengbing Hu, Xudong Lai, Hongwei Chen, Image Segmentation Using Thresholding and Swarm Intelligence, Journal of Software, Vol. 7, NO. 5, 2012.

[13] Chan S., M. K Tiwari, Swarm Intelligence: Focus on Ant and Particle Swarm Optimization, Book edited by: Felix T., ISBN 978-3-902613-09-7, pp. 532, Itech Education and Publishing, Vienna, Austria, 2007.

[14] Guofeng J, Wei Z, Zhengwei Y, Zhiyong H, Yuanjia S, Dongdong W, Gan T, Image Segmentation of Thermal Waving Inspection based on Particle Swarm Optimization Fuzzy Clustering Algorithm, Measurement Science Review, Volume 12, No. 6, 2012.

[15] Sağ T., Çunkaş M. "Development of Image Segmentation Techniques using Swarm IntelligenceABC-Based Clustering Algorithm for Image Segmentation" International Conference on Computing and Information Technology (ICCIT 2012),.pp.95-100, Saudi Arabia,2012.

[16] Fahd M. A. Mohsen, Mohiy M. Hadhoud, Khalid Amin, A new Optimization-Based Image Segmentation method By Particle Swarm Optimization, 10-18. In IJACSAInternational Journal of Advanced Computer Science and Applications, Special Issue on Image Processing and Analysis, 2011

[17] Jevtic, A., Quintanilla-Dominguez, J., Cortina-Januchs, M. G., and Andina, D. Edge detection using ant colony search algorithm and multiscale contrast enhancement. In Proceedings of the 2009 IEEE International Conference on Systems, Man, \& Cybernetics, SMC 2009, pages 2193-2198. 2009.

[18] J. Sun, B. Feng, and W. Xu, (2004) Particle swarm optimization with particles having quantum behavior. In Evolutionary Computation, CEC2004. Congress on. Volume 1, pp 111-116. IEEE.

[19] J. Sun, W. Xu, and B. Feng, (2005) A global search strategy of quantum-behaved particle swarm optimization. In Cybernetics and Intelligent Systems, 2004 IEEE Conference on, volume 1, pp 111-116. IEEE.

[20] J. Kennedy, " The Particle Swarm: Social Adaptation of knowledge", Proceedings of the IEEE International
Conference on Evolutionary Computation, Indianapolis, Indiana, USA, pp. 303-308.

[21] Coelho, L.S. (2007) 'Novel Gaussian quantum-behaved particle swarm optimizer applied to electromagnetic design', Science, Measurement \& Technology, IET, Vol. 1, No. 5, pp. 290-294.

[22] Chandragupta Mauryan, K.S., Thanushkodi K. and Sakthisuganya A., Reactive Power Optimization Using Quantum Particle Swarm Optimization, Journal of Computer Science 8 (10): 1644-1648, 2012.

[23] Leandro dos Santos Coelho, Gaussian quantum-behaved particle swarm optimization approaches for constrained engineering design problems, Journal of Expert Systems with Applications, Vol 37. Issue 2,Pages: 1676-1683, 2010.

[24] Chatterjee A, Mahanti G. K., Comparative Performance of Gravitational Search algorithm and modified particle swarm optimization algorithm for synthesis of thinned scanned concentric ring array antenna, Progress In Electromagnetics Research B, Vol. 25, 331-348, 2010.

[25] Gauci M, Dodd T. J, R. Groß, Why 'GSA: a gravitational search algorithm' is not genuinely based on the law of gravity, journal of Natural computing, ISSN 1572 - 9796, Vol. 11, nº 4, pp. 719-720. 2012.

[26] Rashedi E, Nezamabadi-pour H, Saryazdi S, GSA: a gravitational search algorithm. InfSci 179:2232-2248. 2009.

[27] Ghorbani F., Nezamabadi-pour H., On the Convergence Analysis of Gravitational Search Algorithm, Journal of Advances in Computer Research Quarterly ISSN: 2008 6148. Vol. 3, No.2. Pages: 45-51.2012.

[28] Mirjalili S., MohdHashim S.Z., “A New Hybrid PSOGSA Algorithm for Function Optimization, in IEEE International Conference on Computer and Information Application (ICCIA 2010), China, 2010, pp.374-377. 2010.

[29] Bahrololoum A., Nezamabadi-pour H., Bahrololoum H., Saeed M., "A prototype classifier based on gravitationa search algorithm", Applied Soft Computing, vol. 12, no. 2 , (2012) 819- 825 .

[30] M. A. Potter and K. A. D. Jong, "A cooperative coevolutionary approach to function optimization," in PPSN III: Proceedings of the International Conference on Evolutionary Computation. The Third Conference on Parallel Problem Solving from Nature. London, UK:Springer-Verlag, 1994, pp. 249-257.

[31] Pham. DT., Ghanbarzadeh A, Koc E, Otri S, Rahim S and Zaidi M. :The Bees Algorithm. Technical Note, Manufacturing Engineering Centre, Cardiff University, UK (2005)

[32] Pham DT., Ghanbarzadeh A., Koç E., Otri .S., Rahim .S., Zaidi .M.:The Bees Algorithm - A Novel Tool for Complex Optimisation Problems, Proceedings of IPROMS 2006 Conference, pp.454-461 (2006).

[33] S.Nebti. Color Image Segmentation based on Swarm Optimisation Methods. Lecture Notes in Computer Science, Volume 6377/2010, 277-284, ICICA’2010. 